\title{
Mechanisms involved in treadmill walking improvements in Parkinson's disease
}

\author{
Olalla Bello, Gonzalo Márquez, Miguel Camblor, Miguel Fernández-Del-Olmo
}

\begin{abstract}
Patients with Parkinson's disease (PD) improve gait after treadmill training and while they are walking over the treadmill. However, the mechanisms of these improvements have not been addressed. We designed a treadmill simulator without a belt that could move on a walkway in a constant speed, in order to explore the mechanism underlying treadmill walking improvements in PD. All subjects were tested in three different sessions (treadmill, simulator $_{\text {assisted }}$ and simulator ${ }_{\text {not assisted }}$ ). In each session, subjects first walked overground and then walked using the treadmill or simulator with the hands over the handrails (simulator ${ }_{\text {assisted }}$ ) or with the hands free (simulator ${ }_{\text {not assisted }}$ ). Step length, cadence, double support time, swing time, support time and the coefficient of variation (CV) of step time and double support time were recorded. Over the treadmill PD patients increased their step length and reduced significantly their cadence and $\mathrm{CV}$ of double support time in comparison with overground walking. In the simulator ${ }_{\text {assisted }}$ condition PD patients reduced significantly the $\mathrm{CV}$ of double support time in comparison with overground walking. With the simulator ${ }_{\text {not assisted }}$ both groups decreased their step length and increased their cadence and CV of double support time, compared with walking overground. These findings suggest that the step length improvement observed in PD patients, walking over a treadmill, is due to the proprioceptive information generated by the belt movement, since no improvement was reported when patients using a treadmill simulator.
\end{abstract}

Keywords

Parkinson's disease; Rehabilitation; Gait; Treadmill; Physical therapy

\section{Introduction}

Gait disturbances are one of the principal and most incapacitating symptoms of Parkinson's disease (PD). PD gait is characterized by a decreased stride length with a particular difficulty in its internal regulation [1]. Gait disorders may also include a reduced gait speed, stooped and forward-flexed posture, reduced arm swing, gait instability, falls, and increased stride-to-stride variability [2], [3], [4], [5] and [6].

Only a few studies have investigated the use of treadmill training for gait rehabilitation in PD. The findings show that gait speed and stride length are increased [7], and postural stability is improved [8]. Effects on gait are immediate and are reported after only a single session of treadmill walking [9], [10] and [11]. In addition, advanced PD patients $(H \& Y=3)$ show an adaptation effect with an increase in their step length compared to moderate PD patients or healthy control subjects during treadmill walking [9]. Gait variability is also reduced in PD patients when they are walking over the treadmill [12].

Although there is a growing interest in the therapeutic use of the treadmill walking in PD, little is known regarding the mechanisms involved in gait improvements related to treadmill training. The positive effect of the treadmill in other pathologies, such as spinal cord injury, has been attributed to the activation of the central pattern generator (CPG) [13] and [14]. However, it is undetermined whether this is the mechanism underlying the gait improvements observed in PD patients. The deficits in gait in PD is the result of an impairment in locomotor control due to neurotransmitter imbalance in the basal ganglia [15]. The basal ganglia play an essential role in maintaining movement automaticity [16]. Thus, PD patients cannot properly perform automatic sequential movements, such as walking [17]. However, the ability to generate a normal stepping pattern is not lost in PD and, in contrast with other pathologies, normal stride length can be elicited using external cues or attentional strategies [17]. Thus, it is possible that there are different mechanisms other than the CPG that are responsible for the gait improvements due to treadmill walking in PD patients. For example, walking on the treadmill could provide an external cue that is mediated through proprioceptive receptors [18]. PD patients may benefit from holding the handrails, since balance difficulties and falls are often present in these patients [2] and [19]. The constant 
speed imposed by the treadmill forces the patients to adapt their gait pace to the machine rhythm. In turn this reduces the degrees of freedom and can help to minimize stride-to-stride variations in gait timing [12]. A steady environment can also reduce distractions by focusing attention on the gait, since it is well known that attention plays an important role in PD gait [20]. It has also been suggested that the patients could take advantage of the visual feedback from the front of the treadmill [9] and thus improve their gait.

The main goal of this study was to explore the different mechanisms underlying treadmill walking improvements in Parkinson's disease patients. To this end, we developed a modified treadmill that simulates the treadmill conditions, with the exception that it does not have a belt. This simulator has the same physical characteristics of the treadmill and is able to move with a pre-programed speed.

\section{Methods}

\subsection{Subjects}

Nine patients with PD (eight males and one female) and nine age-, sex-, weight-, leg length- and height-matched controls (eight males and one female) participated in the study. Subjects were recruited from a local Parkinson's Association. PD patients were in the advanced stage (Hoehn and Yahr 3). All the subjects had not walked on a treadmill in the last 12 months prior to the experiment. Subjects were excluded if they had a past history of neurological conditions other than PD or orthopaedic, cardiovascular and visual disturbances that can affect their walking ability. A fundamental requirement for inclusion in the study was the ability to walk for $10 \mathrm{~min}$ without stopping, and without walking aids or assistance. A neurologist examined the level of functional disability of patients using the Unified Parkinson's Disease Rating Scale (UPDRS). No subject showed dementia as assessed by the mini-mental state examination (MMSE). Tests were performed while the patients were ON medication, confirmed by a neurologist evaluating the motor items of the UPRDS scale. Seven out of the nine PD patients had freezing phenomenon only OFF medication and none of the patients had freezing episodes during the experimental trials. All subjects gave informed consent according to the declaration of Helsinki (1964) and the protocol was approved by ethics committee of University of Coruña. Details of PD patients and control subjects are shown in Table 1 and Table 2 , respectively.

Table 1. Details of Parkinson's disease patients characteristics.

\begin{tabular}{|c|c|c|c|c|c|c|c|c|}
\hline $\begin{array}{l}\text { Patient } \\
\text { number }\end{array}$ & $\begin{array}{c}\text { Age } \\
\text { (years) }\end{array}$ & Sex & $\begin{array}{l}\text { Disease } \\
\text { duration } \\
\text { (years) }\end{array}$ & $\begin{array}{l}\text { Weight } \\
(\mathrm{kg})\end{array}$ & $\begin{array}{l}\text { Leg length } \\
\quad(\mathrm{m})\end{array}$ & $\begin{array}{l}\text { Height } \\
\text { (m) }\end{array}$ & $\begin{array}{l}\text { UPDRS } \\
\text { motor }\end{array}$ & Medication per day (mg) \\
\hline 1 & 76 & M & 6 & 85 & 0.90 & 1.65 & 49 & Levodopa/Carbidopa 300/75, Entacapone 600 \\
\hline 2 & 71 & M & 5 & 84 & 0.93 & 1.70 & 40 & $\begin{array}{l}\text { Levodopa/Carbidopa 400/100, Pramipexole 2.1, } \\
\text { Rotigotine } 14\end{array}$ \\
\hline 3 & 80 & M & 20 & 73 & 0.88 & 1.61 & 42 & Levodopa/Carbidopa 1150/175 \\
\hline 4 & 60 & M & 23 & 105 & 1.02 & 1.90 & 47 & $\begin{array}{l}\text { Levodopa/Carbidopa 750/187.5, Ropinirole 4, } \\
\text { Rotigotine } 8\end{array}$ \\
\hline 5 & 72 & M & 4 & 85 & 0.82 & 1.67 & 46 & $\begin{array}{l}\text { Levodopa/Carbidopa 750/187.5, Entacapone } \\
\text { 1000, Cabergoline } 4\end{array}$ \\
\hline 6 & 65 & M & 15 & 99 & 0.91 & 1.69 & 28 & $\begin{array}{l}\text { Levodopa/Carbidopa 500/1250, } \\
\text { Levodopa/Benserazide 500/1250, Entacapone } \\
\text { 1000, Pramipexole } 2.1\end{array}$ \\
\hline 7 & 72 & $\mathrm{~F}$ & 18 & 59 & 0.79 & 1.50 & 28 & $\begin{array}{l}\text { Levodopa/Carbidopa 1000/100, Ropinirole 4, } \\
\text { Amantadine } 100\end{array}$ \\
\hline 8 & 75 & M & 10 & 71 & 0.85 & 1.55 & 41 & $\begin{array}{l}\text { Levodopa/Carbidopa 200/55, } \\
\text { Levodopa/Benserazide 800/200, Ropinirole } 6 \text {, } \\
\text { Rasagiline } 3\end{array}$ \\
\hline 9 & 67 & M & 5 & 70 & 0.85 & 1.64 & 38 & Levodopa/Benserazide 1100/275, Ropinirole 21 \\
\hline Mean & 71 & & 12 & 81 & 0.88 & 1.66 & 38.7 & \\
\hline SD & 6.0 & & 7.7 & 14.4 & 0.07 & 0.11 & 7.3 & \\
\hline
\end{tabular}

\footnotetext{
${ }^{\text {a }}$ Distance from the great trochanter to the floor.
} 
Table 2. Characteristics of control subjects.

\begin{tabular}{lccccc}
\multicolumn{5}{c}{ Control number Age (years) } & Sex Weight (kg) Leg length (m) Height (m) \\
\hline & & & & & \\
1 & 74 & $\mathrm{M}$ & 71 & 0.90 & 1.70 \\
2 & 75 & $\mathrm{~F}$ & 77 & 0.82 & 1.56 \\
3 & 65 & $\mathrm{M}$ & 97 & 0.90 & 1.70 \\
4 & 61 & $\mathrm{M}$ & 74 & 0.84 & 1.69 \\
5 & 59 & $\mathrm{M}$ & 70 & 0.92 & 1.69 \\
6 & 63 & $\mathrm{M}$ & 77 & 0.85 & 1.66 \\
7 & 84 & $\mathrm{M}$ & 83 & 0.89 & 1.63 \\
8 & 76 & $\mathrm{M}$ & 85 & 0.88 & 1.67 \\
9 & 77 & $\mathrm{M}$ & 92 & 0.94 & 1.70 \\
Mean & 71 & & 82 & 0.88 & 1.66 \\
SD & 8.6 & & 9.2 & 0.04 & 0.05 \\
& & & & & \\
\hline
\end{tabular}

\subsection{Apparatus}

\subsubsection{Treadmill and simulator}

A treadmill (SporsArt 6300, Sports Arts Fitness) and a treadmill simulator were used in this study. The treadmill simulator was built by extracting the belt and incorporating four small wheels and a front axle coupled to the motor (Fig. 1). With this modification the treadmill simulator was able to move straightway on a walkway in a fixed speed that was operated by a remote control. This treadmill simulator had the same dimensions of the treadmill. In this way, subjects could walk with the simulator over the ground with the same afferent inputs to that of the treadmill except for the movement of the belt.

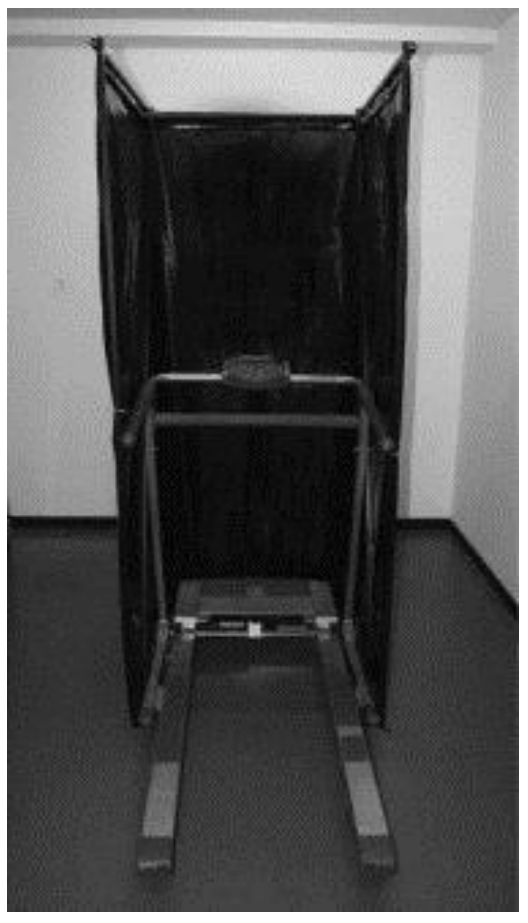

Fig. 1. Posterior view of the simulator. 
Both the treadmill and the simulator were surrounded by a black shade on the front and lateral sides to obtain comparable visual conditions.

The treadmill belt speed was calibrated using previously described methods [21]. The treadmill simulator was calibrated by measuring the time taken for it to complete $15 \mathrm{~m}$ over each of 50 different speeds between 0.27 and $1.6 \mathrm{~m} / \mathrm{s}$, using photoelectric cells. A constant speed for the treadmill simulator across the $15 \mathrm{~m}$ was ensured using a speedometer.

\subsubsection{Gait analysis}

To measure spatiotemporal gait parameters, five foot switches with a diameter of $2 \mathrm{~cm}$ were worn as an insole and placed in each subject's shoe, corresponding to the positions of the heel; base of first, third and fifth metatarsal; and the distal phalanx of the big toe. The foot switches system was attached to a recording unit and synchronized with photoelectric cells.

The variables measured for each condition of gait included: speed $(\mathrm{m} / \mathrm{s})$; step length $(\mathrm{m})$; cadence (steps/min); double support time, swing time and support time (\% of gait cycle); and the coefficient of variation $(\mathrm{CV})$ of step time and double support time (\%). $\mathrm{CV}$ is an indicator of temporal variability of gait, where $\mathrm{CV}=($ standard deviation/mean $) \times 100$. Average step length was calculated by dividing the gait speed by the cadence.

\subsection{Testing procedure}

All subjects were tested in three different sessions (treadmill, simulator ${ }_{\text {assisted }}$ and simulator not assisted $_{\text {) }}$ randomized over three consecutive days. All subjects were wearing the same type of shoes (sport shoes). All the sessions for each patient were performed at the same hour of the day. In each session, subjects were given two practice trials of overground walking and tested for three trials of overground walking on a 25-m walkway. This number of trials was chosen to ensure a minimum amount of gait cycles $(\sim 30$ steps/trial) and at the same time to avoid fatigue in the PD patients. The time taken for each subject to complete the middle $15 \mathrm{~m}$ of the walkway was measured with photoelectric cells. The average speed $(\mathrm{m} / \mathrm{s})$ of the three trials was calculated. Participants were then familiarized with the different machines. The overground speed of each day was used to set the respective machine speed for each subject.

The treadmill test consisted of one block of 3 min of treadmill walking while holding the handrails. During the first minute, belt speed was increased to the overground speed and measurements on the treadmill were taken in the third minute. No condition of walking on the treadmill with hands free was evaluated as this was too difficult for the patients.

Simulator tests consisted of three trials of walking with the simulator with the hands over the handrails ( simulator $_{\text {assisted }}$ ) or with the hands free ( simulator $_{\text {not assisted }}$ ).

Subjects walked during all sessions under the close supervision of a physical therapist and two assistants to prevent falls.

\subsection{Statistic analysis}

A repeated-measures analysis of variance (ANOVA) was performed to compare the gait parameters in the overground conditions across the three sessions and to control for possible changes induced by interday variability.

To determine the changes in gait over the different sessions, a repeated-measures ANOVA was performed with session (treadmill, simulator $_{\text {assisted }}$ and simulator $_{\text {not assisted }}$ ) and condition (overground, machine) as the main factors and group as the between-subjects factor (PD and control).

Post hoc pairwise comparisons were computed using the Bonferroni correction.

All statistical analysis was performed using SPSS (SPSS, Chicago, IL). None of the data violated the normality assumption necessary to conduct parametric statistical tests. $p \leq 0.05$ was considered statistically significant. 


\section{Results}

\subsection{Baseline}

The ANOVA across the sessions for the overground condition showed no main effect for session in any of the variables. There was no session $\times$ group interaction showing that the gait parameters in the overground condition did not change across the different sessions, for neither the controls nor for the PD patients. There was an overall group effect such that, the speed and the step length was significantly larger in the control group compared to the PD patients $\left(F_{(1,16)}=20.62, p<0.0001\right.$ and $F_{(1,16)}=16.01$, $p=0.001$, respectively). The cadence, swing, support and double support times were not significantly different between the two groups. The coefficients of variation of the step time and double support time were significantly larger in PD patients compared to controls $\left(F_{(1,16)}=23.25, p<0.0001\right.$ and $F_{(1,16)}=5.33$, $p=0.035$, respectively). Table 3 shows the means and standard deviations of all the gait variables.

Table 3. Means and standard deviations of gait variables in PD and control groups.

\begin{tabular}{|c|c|c|c|c|c|c|}
\hline \multirow[t]{3}{*}{ Condition } & \multicolumn{6}{|c|}{ Session } \\
\hline & \multicolumn{2}{|c|}{ Treadmill } & \multicolumn{2}{|c|}{ Simulator $_{\text {assisted }}$} & \multicolumn{2}{|c|}{ Simulator ${ }_{\text {not assisted }}$} \\
\hline & Overground & Machine & Overground & Machine & Overground & Machine \\
\hline \multicolumn{7}{|l|}{ PD group } \\
\hline Gait speed (m/s) & $0.79 \pm 0.20$ & & $0.81 \pm 0.20$ & & $0.83 \pm 0.13$ & \\
\hline Step length (m) & $0.47 \pm 0.12$ & $0.54 \pm 0.11^{*}$ & $0.48 \pm 0.12$ & $0.50 \pm 0.14$ & $0.47 \pm 0.12$ & $0.44 \pm 0.12^{*}$ \\
\hline Cadence (steps/min) & $101.46 \pm 14.94$ & $87.63 \pm 12.98^{*}$ & $100.91 \pm 15.30$ & $98.45 \pm 11.95$ & $102.65 \pm 15.00$ & $111.03 \pm 20.03^{*}$ \\
\hline Stance time $(\%)$ & $62.19 \pm 2.65$ & $62.04 \pm 2.45$ & $61.56 \pm 2.77$ & $61.45 \pm 3.36$ & $62.41 \pm 2.86$ & $62.59 \pm 2.34$ \\
\hline Swing time $(\%)$ & $37.83 \pm 2.66$ & $37.95 \pm 2.45$ & $38.41 \pm 2.78$ & $38.53 \pm 3.36$ & $37.62 \pm 2.86$ & $37.40 \pm 2.36$ \\
\hline Double support time $(\%)$ & $24.58 \pm 5.20$ & $24.00 \pm 4.98$ & $24.44 \pm 4.92$ & $24.18 \pm 6.11$ & $25.09 \pm 5.37$ & $25.54 \pm 4.37$ \\
\hline CV of step (\%) & $5.94 \pm 2.47$ & $4.37 \pm 2.17$ & $5.70 \pm 2.93$ & $5.17 \pm 3.50$ & $5.91 \pm 2.96$ & $6.44 \pm 1.81$ \\
\hline $\mathrm{CV}$ of double support $(\%)$ & $9.88 \pm 4.25$ & $6.46 \pm 3.26^{*}$ & $7.74 \pm 1.92$ & $6.27 \pm 1.68^{*}$ & $9.01 \pm 3.88$ & $10.20 \pm 4.06^{*}$ \\
\hline \multicolumn{7}{|l|}{ Control group } \\
\hline Gait speed (m/s) & $1.32 \pm 0.33$ & & $1.33 \pm 0.31$ & & $1.32 \pm 0.29$ & \\
\hline Step length (m) & $0.70 \pm 0.13$ & $0.70 \pm 0.11$ & $0.70 \pm 0.13$ & $0.70 \pm 0.13$ & $0.70 \pm 0.12$ & $0.66 \pm 0.15^{*}$ \\
\hline Cadence (steps/min) & $112.55 \pm 11.58$ & $111.63 \pm 11.53$ & $112.91 \pm 10.31$ & $112.94 \pm 9.97$ & $113.18 \pm 9.75$ & $119.36 \pm 8.71^{*}$ \\
\hline Stance time $(\%)$ & $60.45 \pm 2.05$ & $60.53 \pm 2.23$ & $60.53 \pm 1.75$ & $60.68 \pm 1.51$ & $60.73 \pm 1.82$ & $60.48 \pm 3.14$ \\
\hline Swing time $(\%)$ & $39.55 \pm 2.08$ & $39.47 \pm 2.22$ & $39.48 \pm 1.75$ & $39.33 \pm 1.50$ & $39.26 \pm 1.86$ & $38.48 \pm 2.01$ \\
\hline Double support time $(\%)$ & $20.91 \pm 4.14$ & $21.08 \pm 4.45$ & $21.05 \pm 3.51$ & $21.35 \pm 3.00$ & $21.48 \pm 3.67$ & $21.96 \pm 4.17$ \\
\hline CV of step (\%) & $2.09 \pm 0.48$ & $1.80 \pm 0.71$ & $2.21 \pm 0.81$ & $2.05 \pm 0.67$ & $2.49 \pm 1.37$ & $3.62 \pm 1.49$ \\
\hline $\mathrm{CV}$ of double support $(\%)$ & $6.41 \pm 0.85$ & $6.67 \pm 1.88$ & $6.20 \pm 1.29$ & $6.46 \pm 1.53$ & $7.35 \pm 3.75$ & 10.8 \\
\hline
\end{tabular}

*Significant differences compared with overground walking. 


\subsection{Machine effects}

The ANOVA (session $\times$ condition $\times$ group) had no significant main effects for the variables swing time, support time and double support time. There was a significant main effect for session in the step length $\left(F_{(2,32)}=4.99, \quad p=0.013\right)$ and cadence $\left(F_{(2,32)}=10.67 . \quad p<0.0001\right)$, with a significant session $\times$ condition $\times$ group interaction $\left(F_{(1,32)}=4.97, p=0.028\right.$ and $F_{(2,32)}=4.52, p=0.039$ for step length and cadence, respectively). Fig. 2 summarizes the post hoc tests, which showed that for the PD patients, the treadmill increased significantly their step length $(p<0.0001)$ and reduced significantly their cadence $(p<0.0001)$ in comparison with the overground condition. In the control group there were no significant differences in the step length and cadence between the treadmill and overground conditions. In the simulator ${ }_{\text {assisted }}$ condition there were no differences in the step length and cadence for both groups compared with the overground condition. For the simulator $r_{\text {not assisted }}$ condition in both groups the step length decreased in comparison with the overground condition $(p=0.009$ and $p=0.004$ for PD and control groups, respectively). The cadence was increased significantly in the PD $(p=0.004)$ and control $(p=0.027)$ groups, when comparing the simulator ${ }_{\text {not assisted }}$ whit the overground condition.
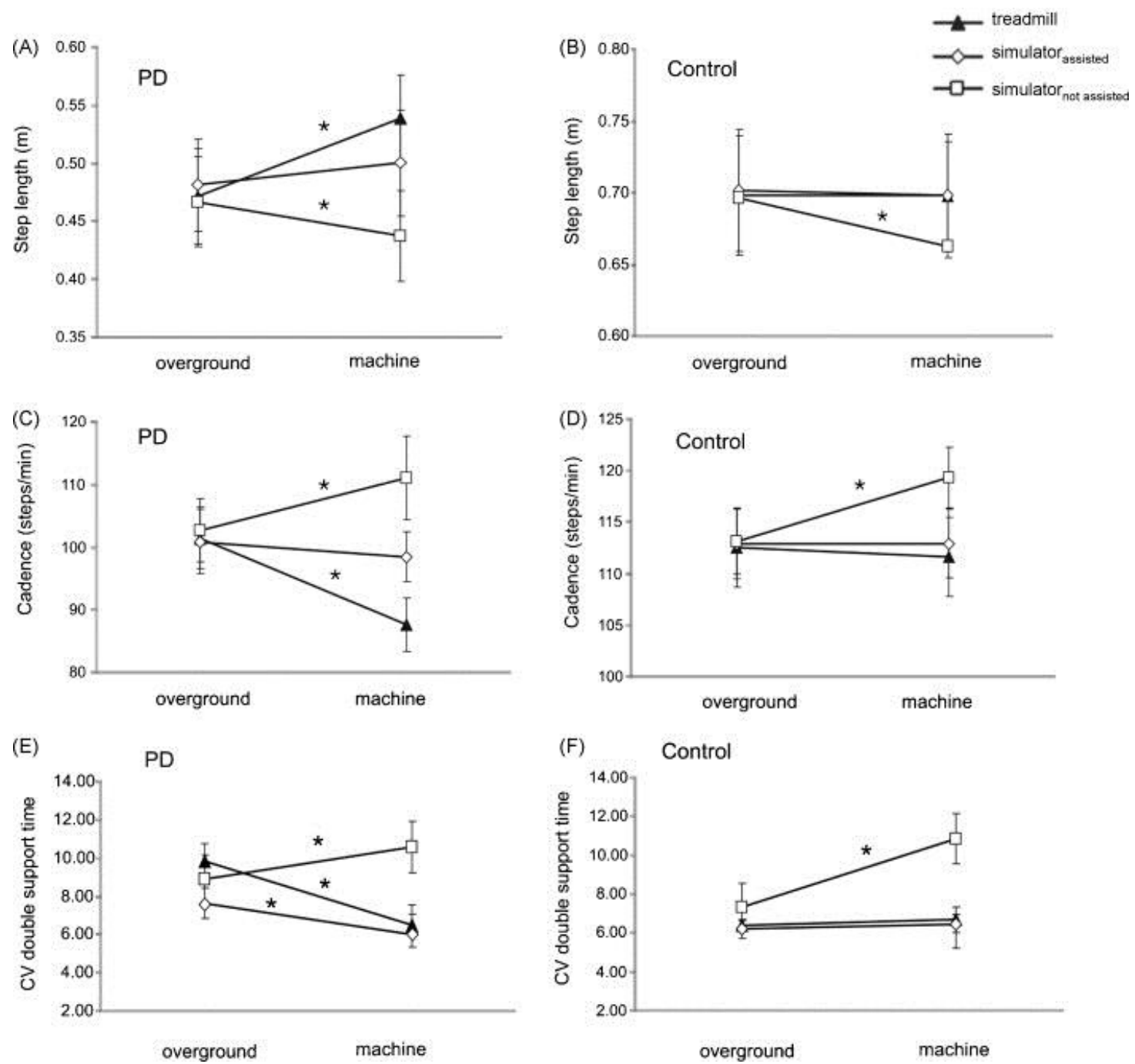

Fig. 2. Kinematic parameters across conditions. Comparison of step length (A, B), cadence (C, D) and coefficient of variation of double support time (E, F) for PD patients and controls for the three different sessions. Note that only the PD patients increased their step length (A) and decreased their cadence (C) over the treadmill in comparison with overground walking. In the simulator not assisted condition both groups decreased their step length and increased their cadence in comparison with overground walking. Only the PD patients decreased their CV (E) over the treadmill and in the simulator ${ }_{\text {assisted }}$ condition in comparison with walking overground. In the simulator ${ }_{\text {not assisted }}$ condition both groups increased their CV. *Significant differences compared with overground walking. 
The ANOVA (session $\times$ condition $\times$ group) had no significant main effect for the CV of step time. However, there was a significant main effect for session for the CV of double support time $\left(F_{(2,32)}=5.33\right.$, $p=0.01)$. For the $\mathrm{CV}$ of double support time there were also significant session $\times$ condition $\left(F_{(2,32)}=10.75, p<0.0001\right)$ and condition $\times$ group $\left(F_{(2,32)}=14.35, p=0.002\right)$ interactions. Post hoc tests showed a significant decrease in the $\mathrm{CV}$ of the double support time over the treadmill $(p=0.007)$ and simulator ${ }_{\text {assisted }}(p=0.025)$ compared with the overground condition for the PD patients but not for the control group (Fig. 2). There was a significant increase in the CV of the double support time in the simulator $_{\text {not assisted }}$ condition compared with overground walking for PD patients $(p=0.05)$ and the control group $(p=0.02)$ (Fig. 2).

\section{Discussion}

\subsection{Group characterization}

The results of the overground conditions are in line with previous studies that showed a decrease in the speed and step length in PD patients compared to controls. These results are constant across the different sessions which indicate that the patients were evaluated in the same medication state. The CVs of step and double support times were always greater in PD than in the control group which is in concordance with the literature [6].

\subsection{Machine effects}

Our results showed that the PD patients improved their step length and reduced the cadence when walking over the treadmill compared with walking overground, replicating previous findings [9]. These results indicate that their walking pattern becomes normalized. No effects were reported for the control group for these parameters when walking over the treadmill. Regarding the coefficients of variation of the step and double support times, we found a decrease in the CV of double support time for PD patients when walking over treadmill and with the simulator ${ }_{\text {assisted }}$ in comparison with overground walking. This improvement of gait variability over the treadmill is in agreement with a previous study that found improvements in the gait variability in PD patients comparing treadmill with overground walking using a walker [12].

Several hypotheses have been proposed to explain the improvements of gait over the treadmill. The first possible set of explanations is related to the visual information provided by the treadmill. It is known that in some conditions visual information may disrupt locomotion and induce freezing (for example passing through a doorway) [22], thus the absence of visual flow when walking on the treadmill could reduce distraction and allow the patients to focus on walking. Another explanation could be that the distance from the front of the treadmill is used as a static visual cue [9]. This cue provides constant visual feedback regarding the position of the body. Thus, the gait can be guided externally rather than internally as in the case of overground walking. However, we propose that these explanations are not the reason for the gait improvements, since we show that PD patients do not improve their step length in both simulator conditions. The simulator and the treadmill both provide the same visual information.

Second, it has been proposed that the constant speed in the treadmill could contribute to minimize stride-to-stride variation in gait timing [12], in contrast with overground walking where there are ongoing fluctuations in gait speed. Our findings show that indeed both the treadmill and simulator ${ }_{\text {assisted }}$ conditions improve the gait in PD patients in comparison with walking overground. However, the PD patients

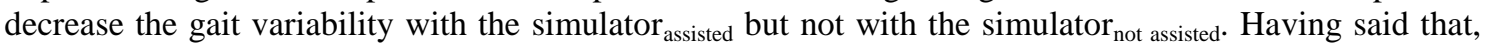

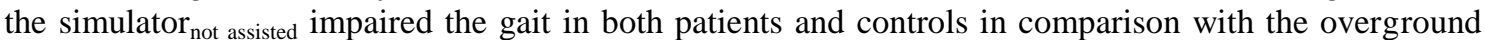
condition, indicating that this condition was more difficult for the subjects to perform. Overall, our findings suggest that PD patients benefit from the imposed constant speed only when they have handrail support. It is important to note that the mechanism underlying the improvement in the gait variability could be different to that of the step length since no improvement in the latter parameter was found in the simulator $_{\text {assisted }}$ condition.

Third, the fact that the patients were able to hold their arms on the handrail of the treadmill improves their balance and may account for the adaptation of the step length observed over the treadmill. It is well known that holding canes and walkers can improve balance and mobility in older adults as well as in patients with different clinical conditions [23]. However, several studies show that PD patients using a walker with wheels decrease speed and stride length [12] and [24]. Our results suggest that holding the arms on the treadmill is not the reason for the step length improvement since in the simulator ${ }_{\text {assisted }}$ 
condition the patients did not improve their step length, despite the fact that they were holding the handrail in the exact way as on the treadmill.

After exclusion of the above hypotheses, our findings suggest that the only possible reason, which may explain the gait improvement in the PD patients over the treadmill, is the belt displacement. The treadmill condition is the only one in which the PD patients increase significantly their step length compared with overground walking and the only difference between the simulator assisted condition and the treadmill is the belt. Thus, the mechanism underling the treadmill improvements in PD patients is due to the proprioceptive information induced by the belt movement. A number of studies have shown improvements in gait during the use of rhythmic auditory or visual sensory cues [17], [25] and [26]. It is thought that in presence of external cues PD patients use a cerebello-premotor pathway [27], alternative to the deteriorated basal ganglia-supplementary motor area pathway. Proprioceptive cues have not been investigated extensively in PD, but if they share the same mechanism of auditory and visual cues, it is possible that they may also be involved in the gait improvement in PD patients. An alternative explanation is that the treadmill belt forces stepping, probably through stretch facilitation of hip flexors and ankle plantar flexors at the end of the stance phase [28]. Therefore, during treadmill walking, PD patients could produce greater hip extensor movements than during overground walking and, thus, increase their stride length. It have been suggested that on the treadmill, the subjects use appropriate sensory inputs such as hip extension that could stimulate the CPG [13] and [29]. However, the relative importance of the CPG in the control of human locomotion remains controversial [30]. Further studies must be conducted in order to contrast these hypotheses.

In summary, our study shows that over the treadmill, the PD patients increase their step length and decrease their cadence compared with overground walking. Overall, our data provide strong evidence that this improvement is related to the belt movement of the treadmill since similar conditions in absence of the belt do not lead to an improvement in gait. Our findings suggest that the gait improvement observed in PD patients walking over a treadmill is due to the proprioceptive information generated by the belt displacement.

\section{Financial disclosure}

This work was supported by Ministerio de Ciencia e Innovación (PSI2008-03175), Spain and Xunta de Galicia (2009/002).

\section{Conflict of interest}

All authors have no conflict of interest and no further financial disclosure to make.

\section{Acknowledgments}

We thank Manuel Iglesias and Jesus Bello for the technical support with the simulator, and Noa Fogelson for the revision of the manuscript.

\section{References}

[1] M.E. Morris, R. Iansek, T.A. Matyas, J.J. Summers The pathogenesis of gait hypokinesia in Parkinson's disease Brain, 117 (5) (1994), pp. 1169-1181

[2] T.A. Boonstra, H. van der Kooij, M. Munneke, B.R. Bloem Gait disorders and balance disturbances in Parkinson's disease: clinical update and pathophysiology Curr Opin Neurol, 21 (4) (2008), pp. 461-471

[3] E. Knutsson An analysis of Parkinsonian gait Brain, 95 (3) (1972), pp. 475-486

[4] M.P. Murray, S.B. Sepic, G.M. Gardner, W.J. Downs Walking patterns of men with parkinsonism Am J Phys Med, 57 (6) (1978), pp. 278-294

[5] W.C. Koller, S. Glatt, B. Vetere-Overfield, R. Hassanein Falls and Parkinson's disease Clin Neuropharmacol, 12 (2) (1989), pp. 98-105

[6] J.M. Hausdorff, M.E. Cudkowicz, R. Firtion, J.Y. Wei, A.L. Goldberger Gait variability and basal ganglia disorders: stride-to-stride variations of gait cycle timing in Parkinson's disease and Huntington's disease Mov Disord, 13 (3) (1998), pp. 428-437

[7] T. Herman, N. Giladi, L. Gruendlinger, J.M. Hausdorff Six weeks of intensive treadmill training improves gait and quality of life in patients with Parkinson's disease: a pilot study Arch Phys Med Rehabil, 88 (9) (2007), pp. $1154-1158$

[8] B.D. Cakit, M. Saracoglu, H. Genc, H.R. Erdem, L. Inan The effects of incremental speed-dependent treadmill training on postural instability and fear of falling in Parkinson's disease Clin Rehabil, 21 (8) (2007), pp. 698-705

[9] O. Bello, J.A. Sanchez, M. Fernandez-del-Olmo Treadmill walking in Parkinson's disease patients: adaptation and generalization effect Mov Disord, 23 (9) (2008), pp. 1243-1249 
[10] I. Miyai, Y. Fujimoto, H. Yamamoto, Y. Ueda, T. Saito, S. Nozaki, et al. Treadmill training with body weight support: its effect on Parkinson's disease Arch Phys Med Rehabil, 81 (7) (2000), pp. 849-852

[11] M. Pohl, G. Rockstroh, S. Ruckriem, G. Mrass, J. Mehrholz Immediate effects of speed-dependent treadmill training on gait parameters in early Parkinson's disease Arch Phys Med Rehabil, 84 (12) (2003), pp. 1760-1766

[12] S. Frenkel-Toledo, N. Giladi, C. Peretz, T. Herman, L. Gruendlinger, J.M. Hausdorff Treadmill walking as an external pacemaker to improve gait rhythm and stability in Parkinson's disease Mov Disord, 20 (9) (2005), pp. $1109-1114$

[13] H.W. Van de Crommert, T. Mulder, J. Duysens Neural control of locomotion: sensory control of the central pattern generator and its relation to treadmill training Gait Posture, 7 (3) (1998), pp. 251-263

[14] V. Dietz, M. Wirz, L. Jensen Locomotion in patients with spinal cord injuries Phys Ther, 77 (5) (1997), pp. 508 516

[15] M.E. Morris, J. McGinley, F. Huxham, J. Collier, R. Iansek Constraints on the kinetic, kinematic and spatiotemporal parameters of gait in Parkinson's disease Hum Mov Sci, 18 (1999), pp. 461-483

[16] R. Iansek, J. Bradshaw, J. Phillips, R. Cunnington, M.E. Morris Interaction of the basal ganglia and supplementary motor area in the elaboration of movement D. Glencross, J. Piek (Eds.), Motor control and sensorimotor integration, Elsevier, Amsterdam (1995), pp. 49-60

[17] M.E. Morris, R. Iansek, T.A. Matyas, J.J. Summers Stride length regulation in Parkinson's disease. Normalization strategies and underlying mechanisms Brain, 119 (2) (1996), pp. 551-568

[18] T. Herman, N. Giladi, J.M. Hausdorff Treadmill training for the treatment of gait disturbances in people with Parkinson's disease: a mini-review J Neural Transm, 116 (3) (2009), pp. 307-318

[19] B.R. Bloem, J.M. Hausdorff, J.E. Visser, N. Giladi Falls and freezing of gait in Parkinson's disease: a review of two interconnected, episodic phenomena Mov Disord, 19 (8) (2004), pp. 871-884

[20] J.M. Hausdorff, J. Balash, N. Giladi Effects of cognitive challenge on gait variability in patients with Parkinson's disease J Geriatr Psychiatry Neurol, 16 (1) (2003), pp. 53-58

[21] A. Matsas, N. Taylor, H. McBurney Knee joint kinematics from familiarised treadmill walking can be generalised to overground walking in young unimpaired subjects Gait Posture, 11 (1) (2000), pp. 46-53

[22] J.P. Azulay, S. Mesure, O. Blin Influence of visual cues on gait in Parkinson's disease: contribution to attention or sensory dependence? J Neurol Sci, 248 (1-2) (2006), pp. 192-195

[23] H. Bateni, B.E. Maki Assistive devices for balance and mobility: benefits, demands, and adverse consequences Arch Phys Med Rehabil, 86 (1) (2005), pp. 134-145

[24] E. Cubo, C.G. Moore, S. Leurgans, C.G. Goetz Wheeled and standard walkers in Parkinson's disease patients with gait freezing Parkinsonism Relat Disord, 10 (1) (2003), pp. 9-14

[25] M.F. del Olmo, J. Cudeiro Temporal variability of gait in Parkinson disease: effects of a rehabilitation programme based on rhythmic sound cues Parkinsonism Relat Disord, 11 (1) (2005), pp. 25-33

[26] M.H. Thaut, G.C. McIntosh, R.R. Rice, R.A. Miller, J. Rathbun, J.M. Brault Rhythmic auditory stimulation in gait training for Parkinson's disease patients Mov Disord, 11 (2) (1996), pp. 193-200

[27] D.J. Brooks Functional imaging studies in Parkinson's disease P. Lewitt, W. Oertel (Eds.), Parkinson's disease. The treatment options, Martin Dunitz, London (1999), pp. 21-38

[28] R. Shepherd, J. Carr Treadmill walking in neurorehabilitation Neurorehabil Neural Repair, 13 (3) (1999), pp. $171-173$

[29] J. Duysens, H.W. Van de Crommert Neural control of locomotion; the central pattern generator from cats to humans Gait Posture, 7 (2) (1998), pp. 131-141

[30] M. MacKay-Lyons Central pattern generation of locomotion: a review of the evidence Phys Ther, 82 (1) (2002), pp. 69-83 